\title{
Investigation of Light Parameters on Image Quality and Optical Coherence Tomography
}

\author{
Boka Fikadu, ${ }^{1}$ Bulcha Bekele, ${ }^{1}$ Leta Tesfaye Jule, ${ }^{1,2}$ Anatol Degefa, ${ }^{3}$ N. Nagaprasad $\left(\mathbb{D},{ }^{4}\right.$ \\ and Krishnaraj Ramaswamy (iD) \\ ${ }^{1}$ Department of Physics, College of Natural and Computational Science, Dambi Dollo University, Dembi Dolo, Ethiopia \\ ${ }^{2}$ Centre for Excellence-Indigenous Knowlegde, Innovative Technology Transfer and Enterpreneurship, Dambi Dollo University, \\ Dembi Dolo, Ethiopia \\ ${ }^{3}$ Department of Mathematics, College of Natural and Computational Science, Dambi Dollo University, Dembi Dolo, Ethiopia \\ ${ }^{4}$ Department of Mechanical Engineering, ULTRA College of Engineering and Technology, Madurai 625 107, Tamil Nadu, India \\ ${ }^{5}$ Department of Mechanical Engineering, College of Engineering, Dambi Dollo University, Dembi Dolo, Ethiopia
}

Correspondence should be addressed to Krishnaraj Ramaswamy; prof.dr.krishnaraj@dadu.edu.et

Received 9 July 2021; Accepted 21 October 2021; Published 26 November 2021

Academic Editor: Wuxiang Zhao

Copyright (C) 2021 Boka Fikadu et al. This is an open access article distributed under the Creative Commons Attribution License, which permits unrestricted use, distribution, and reproduction in any medium, provided the original work is properly cited.

In this work, image quality and optical coherence tomography were studied. The results of the study show that there is a very significant difference between ultrasound and optical coherence tomography to produce an image with a different wave. To understand this, we studied the basic principle of optical coherence tomography in the Michelson interferometer using monochromatic and broadband sources. Time-domain and spectral-domain measurements, which exist at the detector level, are briefly described using a glass sample. The time-domain signal strength of the Michelson interferometer using a broadband source is a Gaussian envelope.

\section{Introduction}

Huang invented optical coherence tomography (OCT) in 1991, and it has since found various applications outside of ophthalmology. For example, OCT is also used to picture certain nontransparent tissues [1]. OCT (optical coherence tomography) system is an optical instrument that provides a cross-sectional image of the biological tissue with an axial resolution of less than 10 microns utilizing light waves [2]. Furthermore, optical coherence tomography (OCT) is a technique that is currently in use in medicine and biology and is capable of creating high-resolution and cross-sectional images of biological tissues [3]. Included in this category are both transparent tissues, including the eyes, and heavily scattering tissues, such as the skin [4]. OCT has very similar applications to ultrasound imaging, but instead of using time-of-flight measurements, OCT utilizes the interference nature of light waves to produce coherence gating [5]. One of the most important components of OCT is that there is no ionizing radiation, and it is not necessary to prepare a sample [6]. Additionally, OCT is comparatively safe when compared with another technique that uses X-rays because it uses light sources without ionizing radiation being involved. Furthermore, with the advantage of being a noninvasive (in vivo) technique, OCT systems show great potential in the future of medical diagnostics. Today, scanning of retinas to reveal defects in the underlying tissue is the area of most success for OCT, and standalone units are being sold commercially [4-6]. The OCT technique is limited to an imaging size of up to $1 \mathrm{~mm}$ below the surface of biological tissues [7]. OCT is specifically good at scanning the retina [8]. Because the retina is readily available to external light, optical coherence tomography (OCT) is particularly useful for diagnosing abnormalities [9]. The light enters the eyes, and the retina converts it into electric signals and passes them to the brain for the formation or reconstruction images $[1,2]$. The major component of the retina is the optics disk, macula, and blood vessels. Using OCT, it is 
easy to see ten different tissue layers inside the retina $[2,5,8]$. Only about a decade ago, the first reports of OCT use in medicine were published in peer-reviewed journals $[1,5,7]$. Although its roots can be traced back to early research on white light interferometry, the discovery of optical coherence domain reflectometry (OCDR), a one-dimensional optical detection technique, is credited with giving rise to the technology [10]. It was initially designed for the purpose of detecting flaws in defective optical fibres and optical network components; nevertheless, its capacity to probe the eye as well as other biological tissues was quickly realized [11]. OCT is a modern variant of a Michelson interferometer, which worked by removing one side of the reference mirror and replacing it with a sample glass to determine the image of the tissue using monochromatic sources and broadband sources [12]. OCT is a type of optical coherence tomography that operates in both the time domain and the spectral domain [13]. For evaluating depth study in time-domain optical coherence tomography, the reference mirror must be moved; however, spectral-domain optical coherence tomography is a Fourier transform analysis performed without adjusting the mirror [2]. In this article, we explain the application of modern optical instruments, specifically for measurements using a Michelson interferometer. Additionally, we simulate the signal Fourier transforms of the materials and compare them with the results of an image of the sample. The primary goal of this study is to figure out the images of OCT using low coherence interference. As a result, time-domain and spectral-domain optical coherence tomography can be determined in broadband sources.

\section{Principle of Optical Coherence Tomography}

OCT has special characteristics, like it is noninvasive and is nondestructive. OCT is used to identify very small structures, and it is used to look under the skin. It can also be used to give information by producing a 3-dimensional image [14].

2.1. Interferences. Young's double-slit experiment is the famous demonstration of optical interference and describes that the light from a single source radiating through two slits (or hole) can interfere with projection of an alternating fringe pattern of bright and dark bands on a screen $[1,6,9]$. These bright and dark fringes occur because of the constructive and destructive interferences of light, respectively. Depending on this bright and dark pattern, the light waves from the two slits are in phase and we add them together up to the resultant maximum amplitude called constructive interference, or when the light waves from the two slits are out of phase, we subtract up to the resultant minimum amplitude called destructive interference. That these two (constructive and destructive interferences) exist are based on the optical path length difference (OPD) between the slits and the same location on the screen $[11,13,15]$.

2.2. Michelson Interferometer. The principle of optical coherence tomography originates with the Michelson interferometer, which was invented by A. A. Michelson in 1881 .
OCT is analogous to ultrasound, as they measure the back reflection intensity of infrared light beams instead of using the echoes of sound. Because of the rapid speed at which light propagates, it is impossible to quantify the back reflection intensity of OCT using electrical instruments [16]. Figure 1 shows a Michelson interferometer, which consists of two reflective mirrors, mirror 1 and mirror 2. Light is emitted from the light source, which hits the beam splitter, with half of the light going along path $L_{1}$ and reflected back to the beam splitter while the other half goes along optical path $L_{2}$ and is reflected back to the beam splitter [17]. These two light waves will interact with each other and be detected with the photodetector. Depending on the length difference between optical paths $L_{1}$ and $L_{2}$, either constructive or destructive interference will occur. Since both return beams will go through the beam splitter (a half-silvered mirror) again, they lose $50 \%$ of their intensity. In some more complicated setups, a polarizing beam splitter (half-silvered mirror) and wave retarders can be used to avoid this power loss.

OCT is a modern instrument that is used to produce an image, and image technology projects a light beam $(820 \mathrm{~nm})$ near the infrared wavelength. Figure 2 shows the setup of OCT, similar to the Michelson interferometer setup. In this setup, only one reference mirror (a movable mirror) is used, and another, the standard mirror, has been changed with a sample of glass. The spectrum from the source of light is separated into different beams using the beam splitter. The probe beam enters the object, and the reference beam approaches the standard mirror at a predetermined distance from the reference mirror. Light reflected from different layers of the specimen is measured and compared to the light reflected from the reference mirror, which measures the echo duration or delay of the light reflected from the reference mirror. When light reflected from both the sample and reference mirrors arrives at the same time, a positive interference is formed. The interferometer integrates numerous data points over a depth of $2 \mathrm{~mm}$ to generate a tomogram of retinal architecture using the information from the data points [18].

From Figure 2, we have an incidence electric field, coefficient of reflection, and transmission of a glass sample. Let us derive the equation of the incident electric field of the sample and calculate the intensity at the detector. Consider the incident light as a plane wave, where the net field at the photodetector includes the electric field coming from $L_{1}$ of the interferometer $E_{0}(t, \tau)=\mathrm{Ee}^{i \omega(t+\tau)}$ and the electric field coming from $L_{2}$ as $E_{0}(t)=\mathrm{Ee}^{i \omega t}$.

\section{Results and Discussion}

In the previous section, we have discussed the principles of OCT and the way OCT can be used like a Michelson interferometer. However, in this case, we review the analysis, discussion, and simulation of this project. The intensity of the Michelson interferometer was simulated, and the signal intensity of the Michelson interferometer in monochromatic and broadband sources was simulated. The signal intensity of OCT in a Michelson interferometer, when one reference 


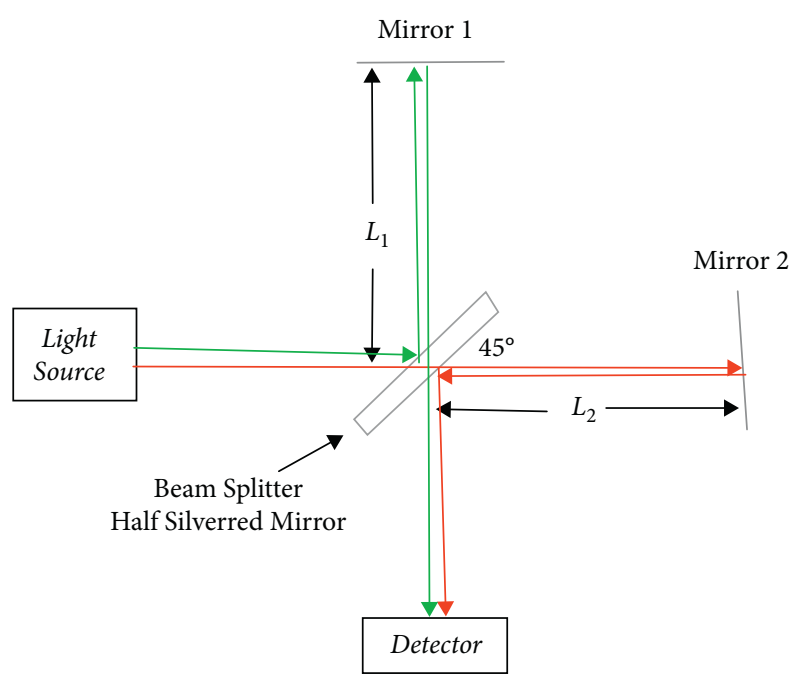

Figure 1: Schematic of the Michelson interferometer.

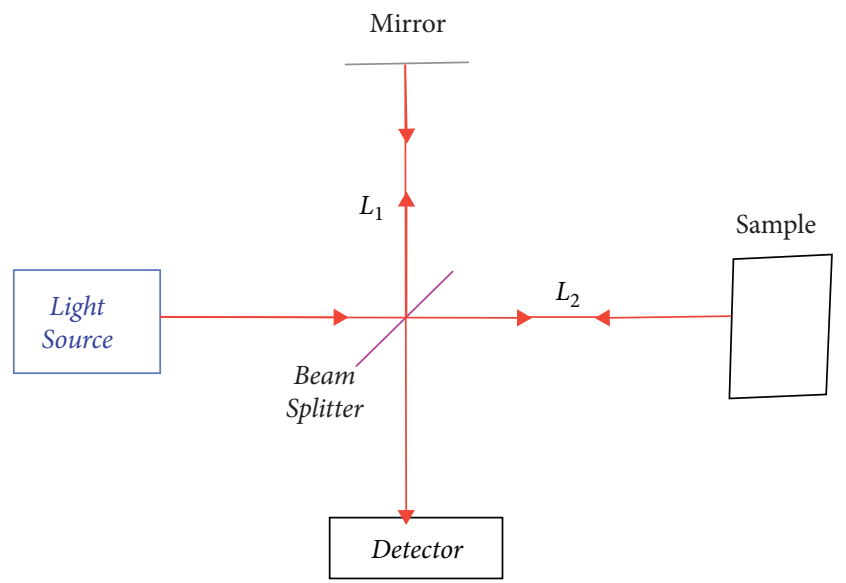

FIGURE 2: Schematic of OCT in Michelson interferometer.

mirror is replaced by a glass sample which has a refractive index of 1.5 and thickness of $1 \mathrm{~mm}$, using monochromatic and broadband sources was simulated [19].

3.1. Simulations of Signal from the Michelson Interferometer. We simulated the intensity with the function of time at a detector. In this simulation, we used the central wavelength $\left(\lambda_{0}\right)$ as $1 \mu \mathrm{m}$ and the spectral width $(\Delta \lambda)$ as $100 \mathrm{~nm}$ for the light source and the velocity and position of the mirror as constant. The intensity which exists at the detector in the Michelson interferometer, with an incident wave as a function of time, is shown in Figure 3.

When the time was zero, the maximum intensity existed and the entire incident light did not occur. We simulated the signal intensity of a monochromatic source, as shown in Figure 4 . The signal intensity of the monochromatic source was simulated, where the horizontal axis shows the time difference between the movable mirror and the fixed mirror. In this simulation, we used the central wavelength $\left(\lambda_{0}\right)$ as $1 \mu \mathrm{m}$ and the spectral width $(\Delta \lambda)$ as $100 \mathrm{~nm}$ for the light source and the velocity and orientation of the mirror as

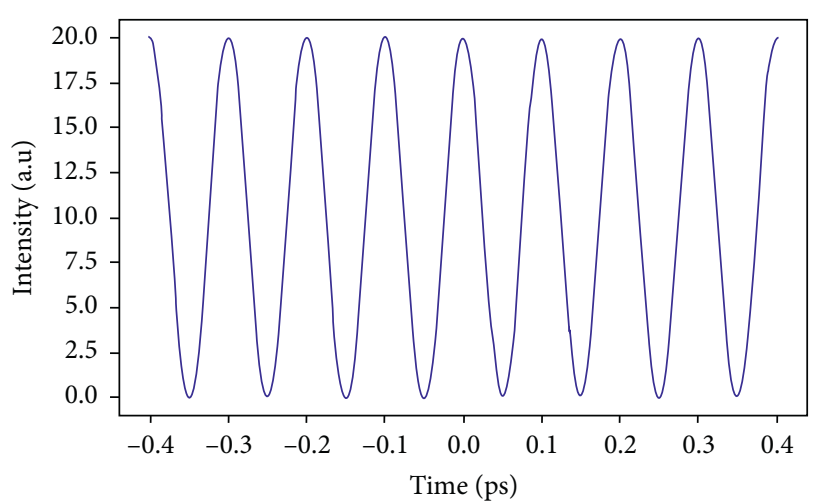

FIgURe 3: Intensity of the Michelson interferometer.

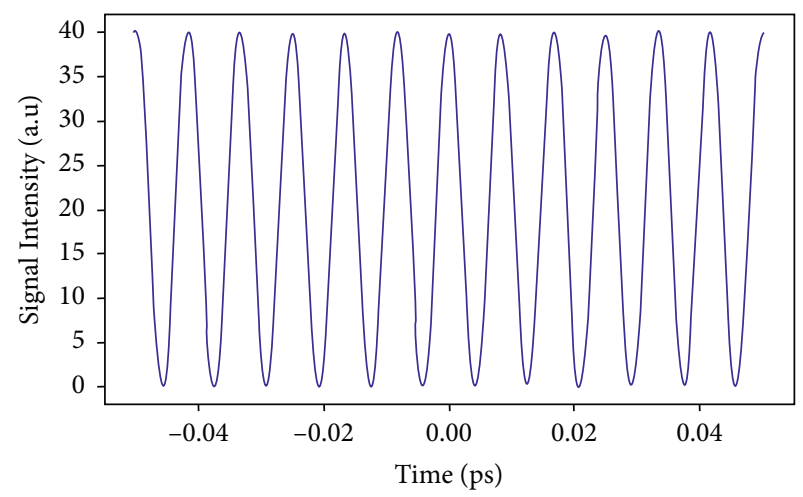

FIgURE 4: Signal intensity of a monochromatic source.

constant. Its greatest possible amplitude refers to constructive interference as well as the minimum amplitude refers to destructive interference. When the time difference is zero, the reflectance is maximum and this is called constructive interference [20].

We simulated the signal intensity of the Michelson interferometer using a broadband coherent source, as shown in Figure 5. This simulation of signal intensity has a Gaussian envelope [21]. In this case, we have some amount of continuous broadband sources, and we used the central wavelength $\left(\lambda_{0}\right)$ as $1 \mu \mathrm{m}$ and the spectral width $(\Delta \lambda)$ as $100 \mathrm{~nm}$ for the light source, where velocity and position of the mirror were constant. For this signal intensity, the movable mirror is of $1.5 \mathrm{ps}$. The horizontal axis shows the time difference of the movable mirror.

From this Michelson interferometer, the signal intensity of light with a Gaussian spectrum as time is either increased or decreased; the output signal intensity started to oscillate. In this case, when we move the reference mirror by halflength, we get a different fringe pattern at each time.

3.2. Simulations of OCT in a Michelson Interferometer. We simulated signal intensity at the detector using a monochromatic source. In this case, one of the reference mirrors is replaced by a sample of glass. This sample glass has a thickness of $1 \mathrm{~mm}$ and a refractive index of 1.5. From the setup, we measure the reflection and transmission of the 


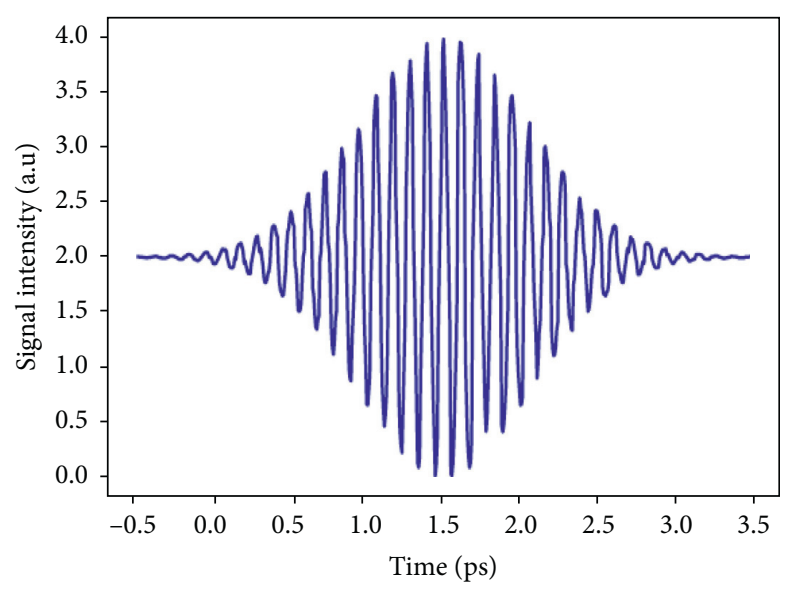

Figure 5: Signal intensity of the broadband coherent source.

sample of glass as a function of time. First, we calculated the electric field, where the Fresnel equations are of perpendicular incidence. That is, there would be a reflection at the first surface and off the second surface and then also multiple reflections, which are called Fabry-Perot reflections [22]. The number of reflections we took into account would depend on the duration of the scan. The signal intensity of OCT in a Michelson interferometer using a monochromatic source with a long coherence length like a variable of time is shown in Figure 6. The maximum amplitude is for constructive interference, and the minimum amplitude is for destructive interference. When the time difference is zero, the reflectance is maximum and it is called constructive interference. This means the monochromatic signal intensity fringes are in phase only for zero time [23-28].

Figure 7 shows the signal intensity of OCT in a Michelson interferometer using a broadband source. The central wavelength of $\left(\lambda_{0}\right) 1 \mu \mathrm{m}$ and the spectral width $(\Delta \lambda)$ of $100 \mathrm{~nm}$ for the light source were simulated. Signal intensity for the optical path length of the sample glass and the movable mirror was matched to the narrow coherence length as the light source used low coherence (broadband sources). The movable mirror started to scan when the optical path length of the reflections from within the sample glass matched each other. The incoming light source was split into two by the beam splitter. This means that when the incoming light passed through two different path lengths $L_{1}$ and $L_{2}$, then it was collinearly superimposed at the beam splitter, the two pulses reflected in each arm of the interferometer, and one of the arm's lengths is increased, the distance causing a delay on recombining. The fundamental wavelength was rejected when the sample glass is detected by a photodetector as a function of the width of the time autocorrelation $=2 \mathrm{nd} / \mathrm{c}$ between the pulses. Since the refractive index and thickness of the glass are 1.5 and $1 \mathrm{~mm}$, respectively, based on these values, we calculated the width of time $=10$ picoseconds $(10 \mathrm{ps})$ and frequency of $1 \times 10^{12}$ Hertz $(1 \mathrm{THz})$. The time delay at the detector using the width of the time pulse was calculated, where the central wavelength was $(\lambda 0) 1 \mu \mathrm{m}$ and the spectral width $(\Delta \lambda)$ was $100 \mathrm{~nm}$, and the result was $3.3 \mathrm{ps}$.

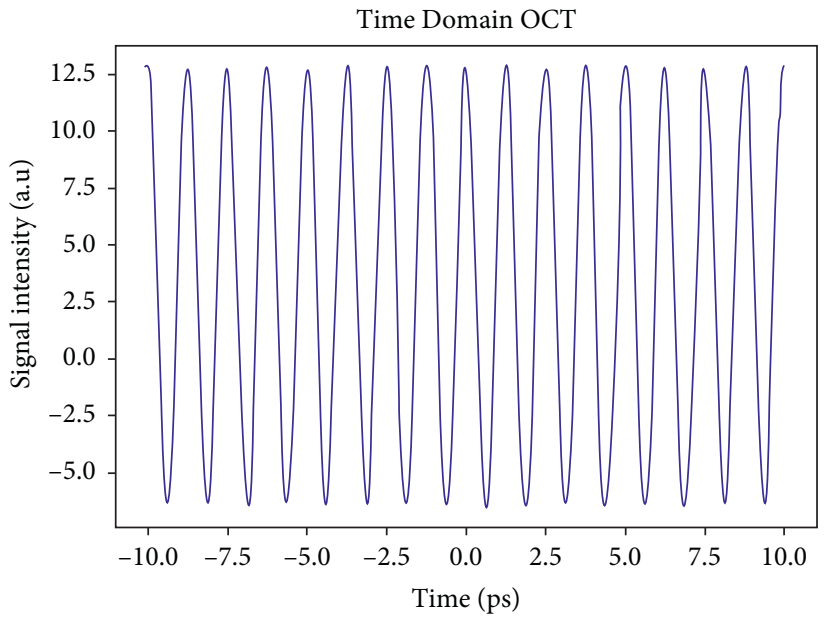

Figure 6: Signal intensity of monochromatic sources.

In this time-domain, OCT, three of these pulses have the same approximate width but different heights. In the simplest method, we measured the shape of the pulse as changing with the distance of the time depth of the reference mirror and then we could see the signal intensity of our sample glass. The time delay of the movable mirror in this signal intensity of OCT was twice the time delay of the movable mirror in the signal intensity of Michelson interferometry. Since we have the values of the refractive index of glass, 1.5 , and the thickness of the glass, $1 \mathrm{~mm}$, we easily simulated the electric field. Additionally, we have some constant values, such as the reflection coefficient and transmission coefficient, as well as the values of the angular frequency $(\omega)$ and tau $(\tau)$, also being constant. After we simulated the electric field, we found the Fourier transform of this electric field. This Fourier transform of the electric field is the spectral domain of the OCT. We measured this spectral domain of the OCT without moving the mirror, and we only focused the Fourier transform of an electric field of a sample glass [29-34]. 

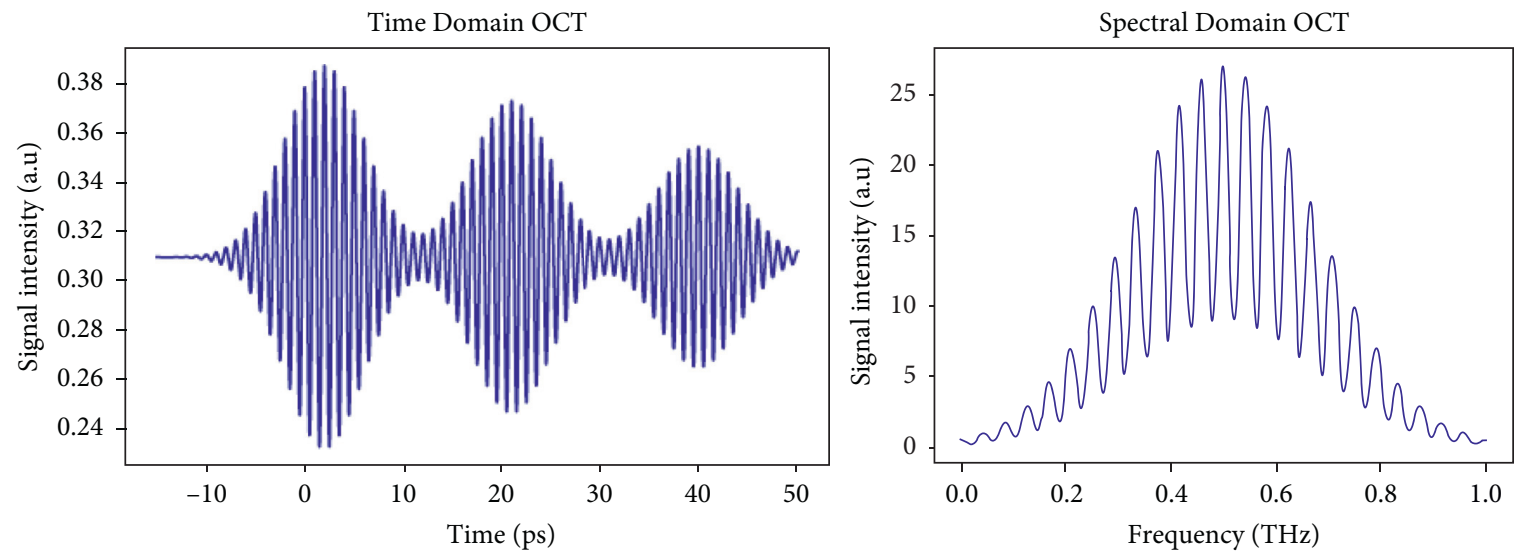

Figure 7: Signal intensity of a broadband coherent source.

\section{Conclusions}

In our work, the application of nanotechnology in integrating OCT with the Michelson interferometer was realized. It is evident that the most important part of an OCT is the time domain and spectral domain. The time domain is based on a moving mirror, but the spectral domain is not based on this moving mirror; it is based on the Fourier transform of an electric field. OCT has many applications in hospitals and industries, but it is mostly used on transparent materials or tissues. OCT is a modern technique that is used to create two- or three-dimensional images without involving any ionizing radiation. The way we measured the signal intensity at the detector in OCT is the same method as for the Michelson interferometer. However, we replaced the fixed mirror with a sample of glass. We then talked about the time domain and spectral domain using a broadband source and a sample glass instead of a reference mirror on one side. We have shown from our results and simulation that the time domain generates ultrafast laser pulses in the range of picoseconds. Some of the characteristics of the pulses were computed, such as their ultrafast laser pulse duration (ps), repetition time, and intensity signal. As our results show, the numerical simulation of using the auto-correlation function to determine the temporal size of ultrafast pulse durations took $10 \mathrm{ps}$ in the time domain of OCT integrated nanotechnology inference was concluded, indicating that the time domain is one of the best techniques for generating ultrafast laser pulses.

\section{Data Availability}

The data used to support the findings of this study are included within the article.

\section{Conflicts of Interest}

The authors declare that there are no conflicts of interest.

\section{References}

[1] M. Tomaniak, D. Ochijewicz, Ł. Kołtowski et al., "OCT-derived plaque morphology and FFR-determined hemodynamic relevance in intermediate coronary stenoses," Journal of Clinical Medicine, vol. 10, no. 11, p. 2379, 2021.

[2] E. A. Rank, R. Sentosa, D. J. Harper et al., "Toward optical coherence tomography on a chip: in vivo three-dimensional human retinal imaging using photonic integrated circuitbased arrayed waveguide gratings," Light: Science \& Applications, vol. 10, pp. 1-15, 2021.

[3] Y. Hamanaka, Y. Sotomi, T. Kobayashi et al., "Comparable neointimal healing in patients with stable coronary lesions and acute coronary syndrome: 3-month optical coherence tomography analysis," The International Journal of Cardiovascular Imaging, vol. 15, pp. 1-11, 2021.

[4] O. Mc Grath, M. W. Sarfraz, A. Gupta, Y. Yang, and T. Aslam, "Clinical utility of artificial intelligence algorithms to enhance wide-field optical coherence tomography angiography images," Journal of Imaging, vol. 7, no. 32, 2021.

[5] J. Zhang, F. Y. Tang, C. Cheung, X. Chen, and H. Chen, "Different effect of media opacity on automated and manual measurement of foveal avascular zone of optical coherence tomography angiographies," British Journal of Ophthalmology, vol. 105, pp. 812-818, 2021.

[6] I. M. Fang, H. Y. Hsu, W. L. Chiang, Y. L. Shih, and C. L. Han, "Correlation between visual acuity and optical coherence tomography angiography parameters in unilateral idiopathic epiretinal membrane," Journal of Clinical Medicine, vol. 10, pp. 26-38, 2021.

[7] M. Dembski, A. Nowinska, K. Ulfik-Dembska, and E. Wylęgała, "Swept source optical coherence tomography analysis of the selected eye's anterior segment parameters," Journal of Clinical Medicine, vol. 10, no. 1094, 2021.

[8] R. Oh, J. Y. Oh, H. J. Choi, M. K. Kim, and C. H. Yoon, "Comparison of ocular biometric measurements in patients with cataract using three swept-source optical coherence tomography devices," BMC Ophthalmology, vol. 21, pp. 1-7, 2021.

[9] Z. Lin, X. Pan, K. Mao et al., "Quantitative evaluation of retinal and choroidal changes in fabry disease using optical coherence tomography angiography," Lasers in Medical Science, 2021.

[10] M. G. Altınel, B. Acikalin, H. Gunes, and G. Demir, "Optical coherence tomography parameters as predictors of treatment response to a 577-nm subthreshold micropulse laser in chronic central serous chorioretinopathy," Lasers in Medical Science, vol. 36, 2021.

[11] A. Miraftabi, S. Jafari, N. Nilforushan, P. Abdolalizadeh, and R. Rakhshan, "Effect of trabeculectomy on optic nerve head 
and macular vessel density: an optical coherence tomography angiography study," International Ophthalmology, vol. 41, 2021.

[12] J. W. Lee, S. Y. Park, P. S. Kim, I. H. Cho, and H. D. Kim, "Correlations among metamorphopsia test scores, optical coherence tomography findings and multifocal electroretinogram responses in epiretinal membrane patients," Documenta Ophthalmologica, vol. 142, no. 3, pp. 293-304, 2021.

[13] S. Gao, Y. Li, D. Bissig et al., "Functional regulation of an outer retina hyporeflective band on optical coherence tomography images," Scientific Reports, vol. 11, pp. 1-13, 2021.

[14] X. Wang, Q. Wei, X. Wu et al., "The vessel density of the superficial retinal capillary plexus as a new biomarker in cerebral small vessel disease: an optical coherence tomography angiography study," Neurological Sciences, vol. 42, 2021.

[15] X. Liu, X. Zhang, J. Ma, and X. Shi, "Integrated system for combined optical coherence tomography-raman spectroscopy of neocaridina denticulate sinensis," Journal of Ocean University of China, vol. 20, no. 1, pp. 94-100, 2021.

[16] S. Cheng, Y. Yu, Y. You et al., "Retinal nerve fiber layer thickness measured by optical coherence tomography predicts visual recovery after orbital decompression for dysthyroid optic neuropathy," International Ophthalmology, vol. 41, 2021.

[17] M. Mohan, S. K. Maurya, K. Kumar, and R. Poddar, "In vitro imaging of animal tissue with upconversion nanoparticles (UCNPs) as a molecular probing agent using swept source optical coherence tomography (SSOCT)," Journal of Medical and Biological Engineering, vol. 40, no. 2, pp. 251-263, 2020.

[18] I. W. Schie, F. Placzek, F. Knorr et al., "Morpho-molecular signal correlation between optical coherence tomography and raman spectroscopy for superior image interpretation and clinical diagnosis," Scientific Reports, vol. 11, pp. 1-14, 2021.

[19] J. Yi, W. Liu, S. Chen et al., "Visible light optical coherence tomography measures retinal oxygen metabolic response to systemic oxygenation," Light: Science \& Applications, vol. 4, no. 9, p. e334, 2015.

[20] T. Zhang, S. Xie, Y. Liu, C. Xue, and W. Zhang, "Effect of amblyopia treatment on macular microvasculature in children with anisometropic amblyopia using optical coherence tomographic angiography," Scientific Reports, vol. 11, pp. 1-7, 2021.

[21] M. F. Alakus, M. Caglayan, N. Ekin et al., "Investigation of corneal topographic and densitometric properties of Wilson's disease patients with or without a Kayser-Fleischer ring," Eye and Vision, vol. 8, pp. 1-8, 2021.

[22] M. Nassisi, C. Lavia, S. Mohand-Said et al., "Near-infrared fundus autofluorescence alterations correlate with sweptsource optical coherence tomography angiography findings in patients with retinitis pigmentosa," Scientific Reports, vol. 11, pp. 1-12, 2021.

[23] L. Barbano, L. Ziccardi, and V. Parisi, "Correlations between visual morphological, electrophysiological, and acuity changes in chronic non-arteritic ischemic optic neuropathy," Graefe's Archive for Clinical and Experimental Ophthalmology, vol. 259, no. 5, pp. 1297-1308, 2021.

[24] C. Zhuo, B. Xiao, C. Chen et al., "Abberant inverted U-shaped brain pattern and trait-related retinal impairment in schizophrenia patients with combined auditory and visual hallucinations: a pilot study," Brain imaging and behavior, vol. 15, no. 2, pp. 738-747, 2021.

[25] S. Abel, J. L. Tesfaye, N. Nagaprasad, R. Shanmugam, L. P. Dwarampudi, and R. Krishnaraj, "Synthesis and characterization of zinc oxide nanoparticles using moringa leaf extract," Journal of Nanomaterials, vol. 2021, Article ID 4525770, 2021.

[26] D. C. Lekha, R. Shanmugam, K. Madhuri et al., "Review on silver nanoparticle synthesis method, antibacterial activity, drug delivery vehicles, and toxicity pathways: recent advances and future aspects," Journal of Nanomaterials, vol. 2021, Article ID 4401829, 2021.

[27] A. Degefa, B. Bulcha, L. T. Jule et al., "Green synthesis, characterization of zinc oxide nanoparticles and examination of properties for dye sensitive solar cells using various vegetable extracts," Journal of Nanomaterials, vol. 2021, Article ID 3941923, 2021.

[28] S. Abel, J. L. Tesfaye, B. Fikadu et al., "Preparation of nano sheets from titanium dioxide nanoparticles synthesized by chemical bath deposition techniques and applications in desalination and waste water treatment," Journal of Nanomaterials, vol. 2021, Article ID 3039761, 2021.

[29] B. Bulcha, J. L. Jule, A. Degefa et al., "Synthesis of zinc oxide nanoparticles by hydrothermal methods and spectroscopic investigation of ultraviolet radiation protective properties," Journal of Nanomaterials, vol. 2021, Article ID 8617290, 2021.

[30] B. Bulcha, A. Degefa, F. Tesgera et al., "Green versus chemical precipitation methods of preparing zinc oxide nanoparticles and investigation of antimicrobial properties," Journal of Nanomaterials, vol. 2021, Article ID 9210817, 2021.

[31] S. Abel, J. L. Tesfaye, R. Shanmugam et al., "Green synthesis and characterizations of zinc oxide $(\mathrm{ZnO})$ nanoparticles using aqueous leaf extracts of coffee (coffea arabica) and its application in environmental toxicity reduction," Journal of Nanomaterials, vol. 2021, Article ID 3413350, 2021.

[32] L. Tesfaye Jule, K. Ramaswamy, B. Bekele, A. Saka, and N. Nagaprasad, "Experimental investigation on the impacts of annealing temperatures on titanium dioxide nanoparticles structure, size and optical properties synthesized through solgel methods," Materials Today: Proceedings, vol. 45, pp. 5752-5758, 2021.

[33] L. T. Jule, R. Krishnaraj, N. Nagaprasad, B. Stalin, V. Vignesh, and T. Amuthan, "Evaluate the structural and thermal analysisof solid and cross drilled rotor by using finite element analysis," Materials Today: Proceedings, vol. 47, 2021.

[34] T. Amuthan, N. Nagaprasad, R. Krishnaraj, V. Narasimharaj, B. Stalin, and V. Vignesh, "Experimental study of mechanical properties of AA6061 and AA7075 alloy joints using friction stir welding," Materials Today: Proceedings, vol. 46, 2021. 